\title{
Primary skeletal muscle tuberculosis in an immunocompetent patient
}

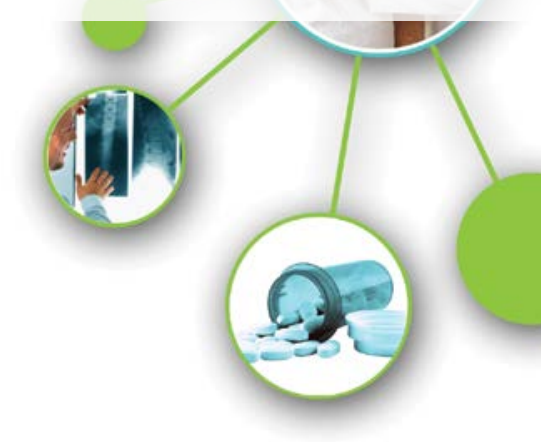

Tuberculosis of soft tissues is rare. We report an exceptional case of tuberculosis in a 42-year-old female. The focus of infection was situated in the anterior muscle compartment of the right thigh. Outcome was good after surgery and a 9-month antituberculosis treatment, with no general or functional sequelae.

\section{KEYWORDS: skeletal muscle, extrapulmonary tuberculosis, immunocompetent}

\section{Introduction}

Tuberculosis can involve virtually any organ and it manifests itself in various forms. Muscular tuberculosis (MT) is a rare localization of the disease $(0.01$ to $2 \%)$. Its occurrence without associated bone involvement is exceptional. MT often simulates a muscular tumor. MRI contributes to the improvement of the sensitivity of the diagnosis of MT because it allows to specify the extent of the muscular lesions and to orient the site of the muscular biopsy. The diagnosis is essentially based on histology [1]. We report the case of isolated tuberculosis of the iliac muscles and quadriceps of the right thigh without any evident primary focus, revealed as an intramuscular mass in a 42-year old immunocompetent female.

\section{Case report}

It is a 42-year-old female, non-smoking, with an unremarkable medical history, and without drug treatment, presented with a 3 -month history of pain and swelling of the antero-internal surface of the right to thigh with impairment of motricity of the right lower limb.

Examination revealed that the patient was afebrile, well nourished, and in no acute distress. Systemic examination findings were unremarkable except for a firm, non mobile and tender soft-tissue mass of $20 \times 15 \mathrm{~cm}$ on the proximal part of the right thigh. The overlying skin appeared normal, with no wounds, rash, or sinuses. It's painful on palpation. The joints above and below were without anomalies. There is no satellite lymph node. Both the biochemical and the tuberculin skin test were negative. HIV serology was negative.

Chest X-ray was within normal limits. X-ray of the pelvis and the right hip (anteroposterior and lateral views) (FIGURE 1) showed a thickening of the soft parts of the thigh without bone lesion. HIV serology was negative.

Magnetic resonance imaging (MRI) showed multiple cystic formations partitioned belong the iliac muscle and the quadriceps in right thigh (FIGURE 2).

Because of high suspicion of tumor we decided to undertake exploration of the swelling/ tumor and decided to perform surgical excision.

Surgery was performed under general anesthesia (smith Paterson incision) with evacuation of 1 liter of purulent fluid. The bacteriological examination was stained for acid fast bacilli on direct microscopy and later culture on Lowenstein Johnson medium was also found to be positive. There were no other tuberculous sites (chest X-ray (FIGURE 3) and BK test by gastric tubing were negative). The patient was treated with antituberculosis drugs for 9 months with good clinical outcome: extinction of pain 15 days after and complete resorption of the mass.

\section{Discussion}

Despite the introduction of tuberculosis

\section{S Daboussi*, \\ S M'hamdi, \\ C Aichaouia, \\ Z Moatamri, \\ M Khadraoui, \\ R Cheikh}

Department of Respiratory Medicine, Military Hospital of Instruction of Tunis

*Author for correspondence:

selsabil_d@yahoo.fr 

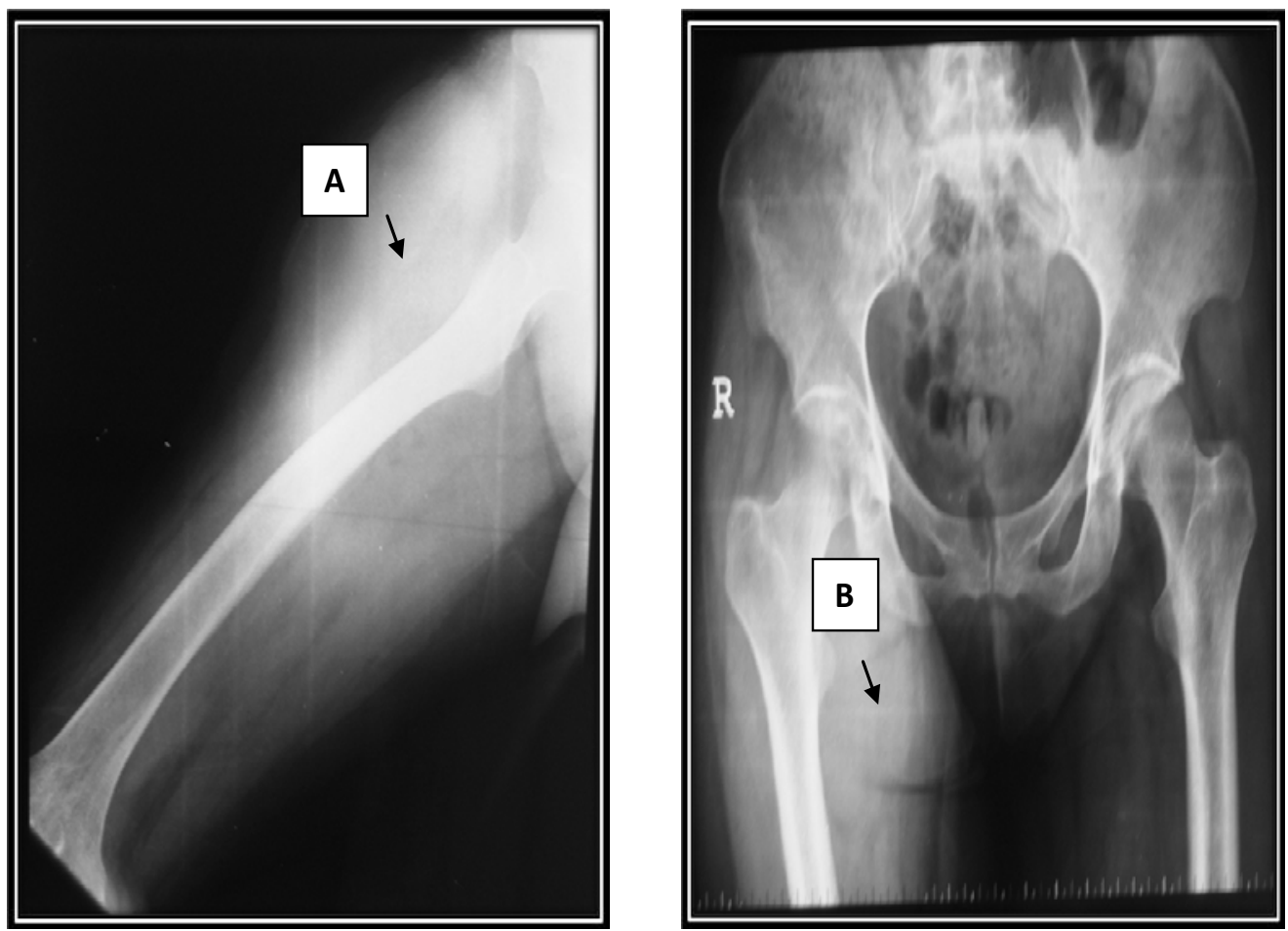

FIGURE 1. Panel A: X-ray of right hip of profile showing a thickening of the soft parts of the right thigh without bone lesion.

Panel B: X-ray of the pelvis: showing a thickening of the soft parts without bone lesion.

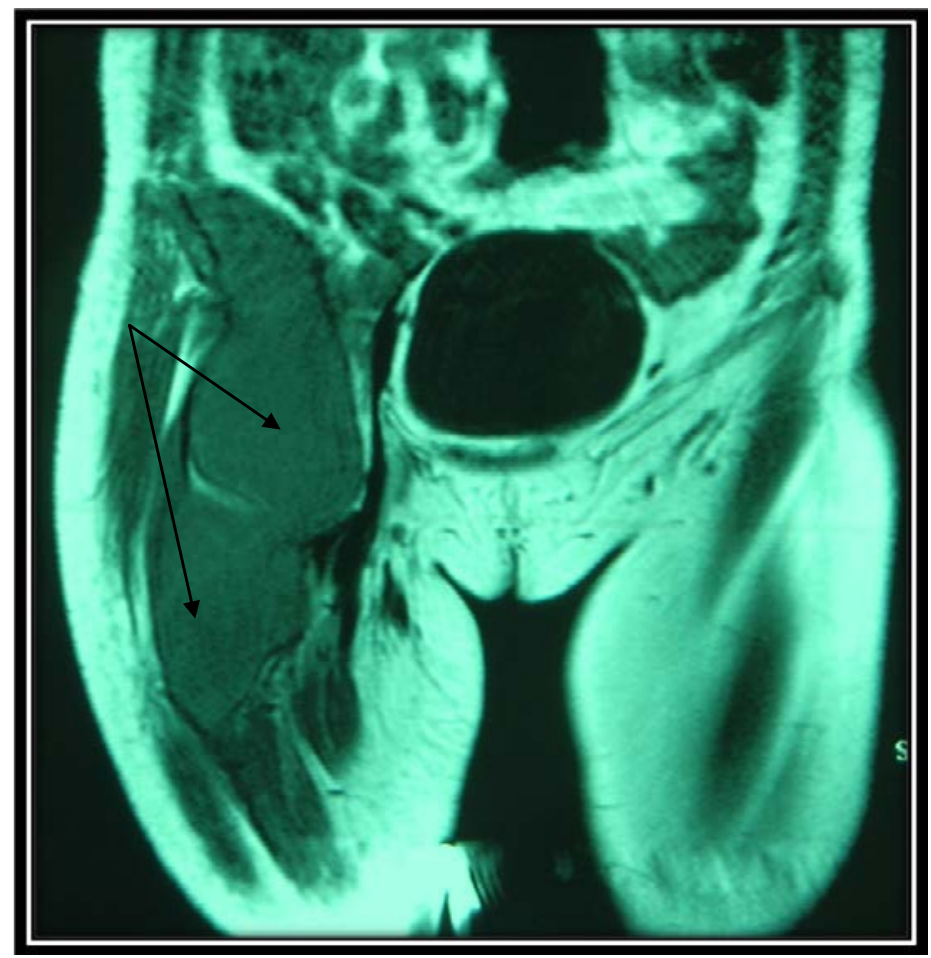

FIGURE 2. Magnetic resonance imaging (MRI): multiple cystic formations partitioned belong the iliac muscle and the quadriceps in right thigh.

treatment and the introduction of tuberculosis vaccination campaigns for several decades, tuberculosis is still a public health problem. In Tunisia, there has been a resurgence of the disease in recent years, with the reappearance of serious and deadly forms. Mycobacterium tuberculosis generally reaches the first to lungs first and then spreads through dissemination. 


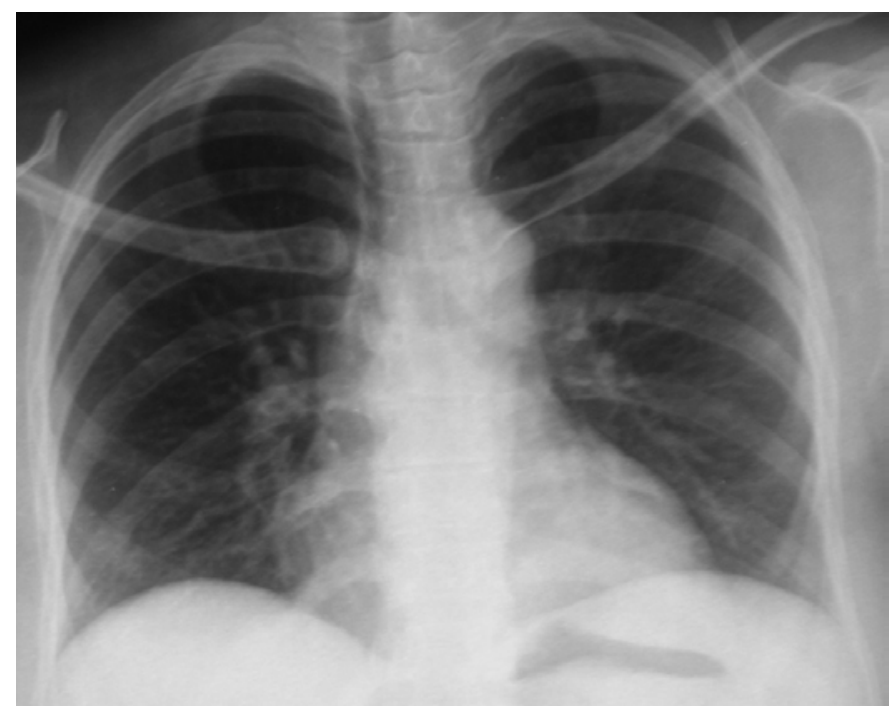

FIGURE 3. Chest X-ray: without abnormalities.

The involvement of the muscles coexists with pulmonary involvement in $30 \%$ of cases [2].

Pulmonary tuberculosis can be associated with rare locations such as tenosynovial or bursit tuberculosis,but in both cases, mycobacterium tuberculosis was unusually isolated $[3,4]$.

The muscle is most often contaminated by a direct extension from a neighboring joint or rarely by haematogenous spread, as is the case with tuberculous spondylitis (psoas abscess) [5].

Musculoskeletal tuberculosis has been reported only sporadically and is mostly due to direct inoculation (intra-muscular injections by infected syringes). The published cases concern mainly children [6-8].

In our patient, no primary localization was found and no notion of iatrogenic inoculation was noted. However, contamination could have been secondary to infraclinical pulmonary tuberculosis.

The rarity of skeletal muscle tuberculosis has been attributed to various factors including high lactic acid content of these muscles, absence of reticuloendothelial/ lymphatic tissue, rich blood supply and highly differentiated state of muscle tissue [9]. Clinical symptomatology associates pain and swelling of the affected muscle. Other constitutional symptoms of tuberculosis may or may not be present. The diagnosis of skeletal muscle tuberculosis remains a challenge for clinicians and requires a very high index of suspicion. The combination of clinical tests with radiological and cytopathological imagery, and a positive tuberculin skin test, provide strong clues pointing to a diagnosis of skeletal muscle tuberculosis. High degree of suspicion is needed for early diagnosis to avoid complications especially in endemic areas [10,11]. In most cases, diagnosis is made months to years after the onset of symptoms: in our patient more than 3 months elapsed. The gold standard for diagnosis is histology, with mandatory microbiological confirmation from the aspirated/surgical material

The treatment is based on a well-conducted antibiotic anti bacillary and prolonged administration of a minimum of four drugs to which the organisms are susceptible. Isoniazid, rifampin, pyrazinamide and ethambutol regimen is used. Operative intervention is an adjunct to appropriate antituberculosis chemotherapy [12]. The optimum duration of treatment has been an issue of considerable debate; the short-course regimens for six months may not be applicable to extrapulmonary tuberculosis, specifically those with osseous involvement. The treatment should be continued for a minimum of nine months [12].

\section{Conclusion}

Muscle tuberculosis remains exceptional and its exact pathogenesis is not yet clearly explained. It should also be considered in the differential diagnosis of painful soft tissue swellings especially in people born in tubercular endemic areas. The prognosis is good if welltreated. 


\section{REFERENCES}

Petter CK. Some thoughts on tuberculosis of fascia and muscle. Lancet 57,156-159 (1937).

Goldblatt M, Cremin BJ. Osteoarticular tuberculosis, its presentation in coloured rades. Clin. Radiol. 29, 669-677 (1978).

Ream-Graves S, Weinstein AJ, Calabrese LH, et al. Tuberculosis of the greater trochanteric bursa. Arthrit. Rheuma. 26,77-81 (1983).

Abdelwahab IE, Kenan S, Hermann G, Kleinmj. Tuberculosis gluteal abcess without bone involvement. Skel. Radiol. 27, 36-39 (1998).
Chapman M, Murray RO, Stoker DJ. Tuberculosis of the bones and joints. Sem. Roentgenol. 14, 266-282 (1979).

Heycock JB, Noble TC. Four cases of syringe-transmitted tuberculosis. Tubercl. 42, 25-27 (1961).

Tamura M, Ogawa G, Sagana I, Amano S. Observations on epidemic of cutaneous and lymphatic tuberculosis which followed use of antityphoid vaccine. Am. Rw. Tuberc. 11, 465-472 (1955).

Robert S, Derkash M, John T. Isolated tuberculosis of the triceps muscle. J. Bone Joint Surg. Am. 61,948 (1979).

Plummer WW, Sanes S, Smith WS, et al. Muscle Tuberculosis. J. Bone Joint Surg. 16,631 (1934).
Wang JY, Lee LN, Hsueh PR, et al. Tuberculous myositis: a rare but existing clinical entity. Rheumatology 42, 836-840 (2003).

Serhan E, Newton P, FitzGerald R, Jassim I. A visible and palpable cause of backache. Ann. Rheum. Dis. 59, 164-165 (2000).

Treatment of Tuberculosis-Guidelines for national programmes. 3rd ed. World Health Organisation, pp 36-38 (2003). 